\title{
Systemic Sclerosis (Scleroderma) with Hypothyroidism in a Four and Half Year Old Child: A Case Report
}

\author{
SA RAHMAN ${ }^{\mathrm{a}}$, J SULTANA ${ }^{\mathrm{b}}$, SS HUQUEc, CA KAWSER ${ }^{\mathrm{d}}$
}

\section{Summary:}

A four and half years old boy was admitted in the Paediatric ward of Bangabandhu Sheikh Mujib Medical University (BSMMU) with the complaints of tightening of skin at different parts of the body and contracture of the joints. Three years back he was admitted in the same hospital for weakness, less activity and constipation. At that time he was diagnosed as a case of hypothyroidism.

\section{Introduction}

Scleroderma is a chronic disease of unknown aetiology that affects the microvasculature and loose connective tissue. It is characterized by fibrosis and obliteration of vessels in the skin and visceral organs including lung, gastrointestinal tract, kidneys and heart. ${ }^{1}$ Scleroderma may occur in a localized form or as a systemic disease. Systemic scleroderma is subdivided by the extent of the skin disease into diffuse cutaneous systemic sclerosis (DCSS), formerly known as progressive systemic sclerosis and limited cutaneous systemic scleroderma. ${ }^{2}$

Scleroderma is a rare disease. ${ }^{3}$ Estimated annual incidence was 4.5 to14 per million. ${ }^{4}$ More recent studies reported the prevalence as 19 to 75 per 100,000 population ${ }^{5}$. Childhood onset is extremely rare: children below 10 years represent fewer than $2 \%$ of all cases, and it has been estimated that less than $10 \%$ of all patients develop systemic sclerosis before

a. Prof. Shahana Akhter Rahman, FCPS (Paediatrics), Professor, Department of Paediatrics, Bangabandhu Sheikh Mujib Medical University

b. Major Jesmin Sultana, FCPS (Paediatrics), Child Specialist, $\mathrm{CMH}$, Dhaka

c. Dr. Syed Saimul Huque, MBBS, Resident Medical Officer, Department of Paediatrics, Bangabandhu Sheikh Mujib Medical University

d. Prof. Choudhury Ali Kawser, FCPS (Paediatrics), PhD, Professor, Department of Paediatrics, Bangabandhu Sheikh Mujib Medical University

Address of Correspondence: Prof. Shahana Akhter Rahman, FCPS (Paediatrics), Professor, Department of Paediatrics, Bangabandhu Sheikh Mujib Medical University, Dhaka..

Received: 9 July, 2006

Accepted: 14 November, 2006
On examination the boy had expressionless face, pinched nose and microstomia. There was tightening and hardening of skin all over the body associated with sclerodactyly and contractures and flexion deformities of all the distal joints of the extremities. From the history, clinical findings and supportive investigations the boy was diagnosed as a case of systemic sclerosis with hypothyroidism.

(J Bangladesh Coll Phys Surg 2007; 25 : 47-51)

the age of 20 years. ${ }^{6}$ Localized scleroderma is more common in children than systemic sclerosis. ${ }^{3}$ There are a number of small series of children with DCSS and a number of case reports totaling just over 100 patients, although many unreported cases may be present ${ }^{4}$. Hypothyroidism is a frequent association with DCSS. It is estimated that about half of the DCSS patients experience overt or latent hypothyroidism. ${ }^{7}$ Because of the rarity of the problem in children, we find it of academic interest to report a case of diffuse cutaneous systemic sclerosis (DCSS) with hypothyroidism, who was admitted in the paediatric ward of Bangabandhu Sheikh Mujib Medical University.

\section{Case Report}

$\mathrm{MH}$, a $4 \frac{1}{2}$ years old boy born to a non-consanguineous parents from Dhaka was admitted with the complaints of gradual development of thickening followed by tightening of skin at different parts of the body for last $2 \frac{1}{2}$ years. Initially there were swollen oedematous areas around the fingers and on the dorsum of the hands and feet. Gradually the swollen areas were thickened and eventually swelling and thickening disappeared and was replaced by tightening of the skin. It gradually progressed all over the body involving face, extremities and trunk. Subsequently there was development of stiffness and deformities of all the small joints and distal large joints of the extremities. MH also developed atrophic depigmentation of skin over the face, chest, abdomen and extremities with loss of hairs from those areas. 
There was history of numbness, pallor and increased contractures of the hand on exposure to cold. MH had no history of arthritis, chest pain, dyspnoea, haematuria or epigastric pain. But there was history of dysphagia, which improved after taking current medicine. His bladder and bowel habits were normal.

MH was admitted in a hospital 3 years back for weakness, less activity and constipation. Laboratory studies at that time were consistent with hypothyroidism having low $\mathrm{T}_{3}$ and $\mathrm{T}_{4}$ levels and high TSH level. Since then he was on thyroxin replacement therapy.

MH was mildly pale and severely wasted (weight for height Z score: -3.5). Vital signs were normal. His face was expressionless with loss of wrinkles, pinched nose, microstomia and puckering of the lips (Fig-1). There were dry, hairless, de-pigmented areas all over the body which were tightly bound to underlying structures and at the same time there were

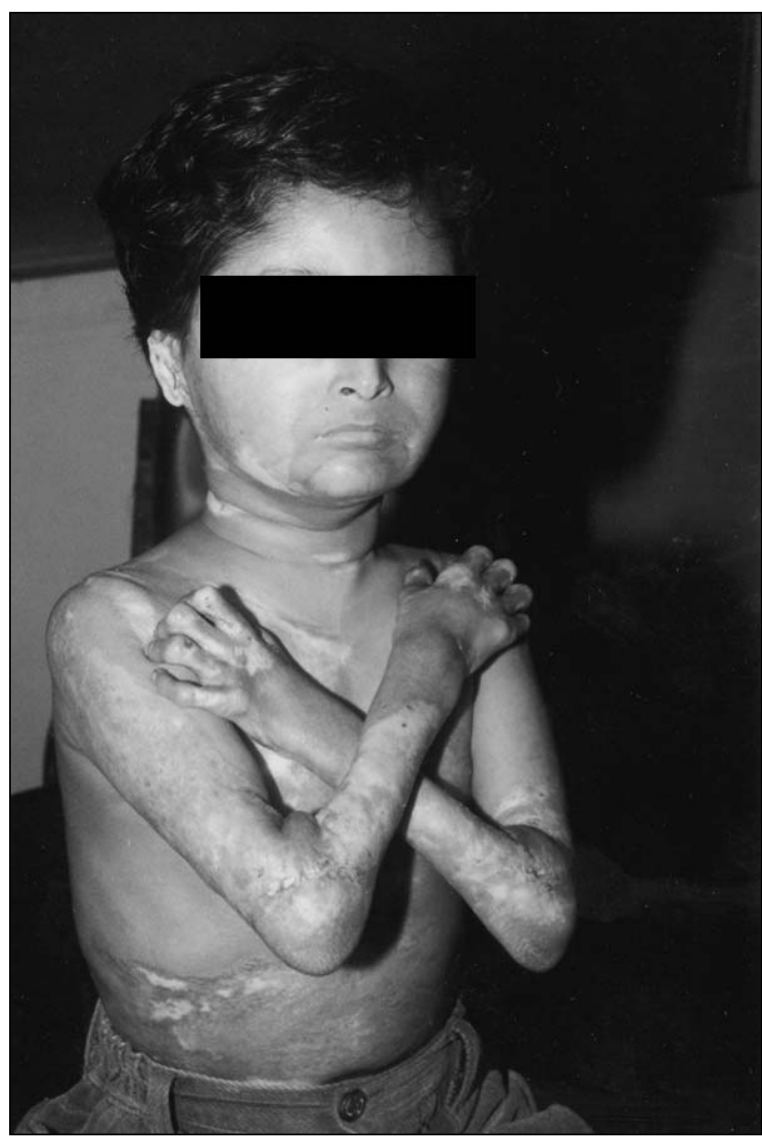

Fig.-1: Expressionless face with loss of wrinkles, pinched nose and microstomia some ulcerated areas as well (Fig-2). All the small joints of the hands and some large joints (wrist, elbow, ankle and knee joints) had severe flexion deformities and gross limitation of movements (Fig2). Fingers of both hands had tapered appearance with sclerodactyly (Fig-3). There were no features of arthritis and respiratory distress. Examinations of other systems were normal.

Investigations showed normal total and differential counts with mild anaemia (Haemoglobin: $10 \mathrm{gm} / \mathrm{dl}$ ) and normal ESR (20 $\mathrm{mm}$ in the $1^{\text {st }}$ hour). Chest x-ray was normal. Renal function, liver function and serum CPK levels were also normal. Free T4 and TSH levels were normal (the child was on replacement therapy). Anti scl-70, anti-thyroid peroxidase and antithyroglobulin antibodies were absent. Ultrasonography of KUB region was normal. DTPA scan showed

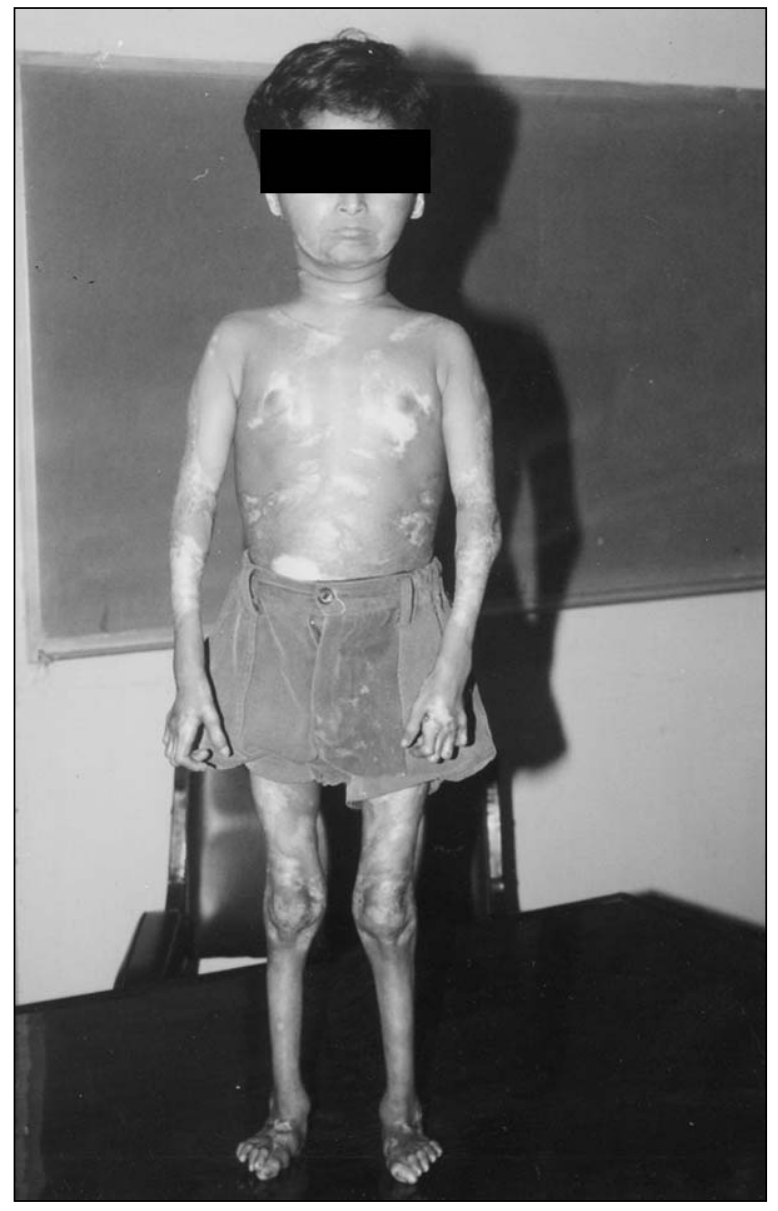

Fig.-2: Dry, hairless de-pigmented areas tightly bound with underlying structures and severe flexion deformities of joints 


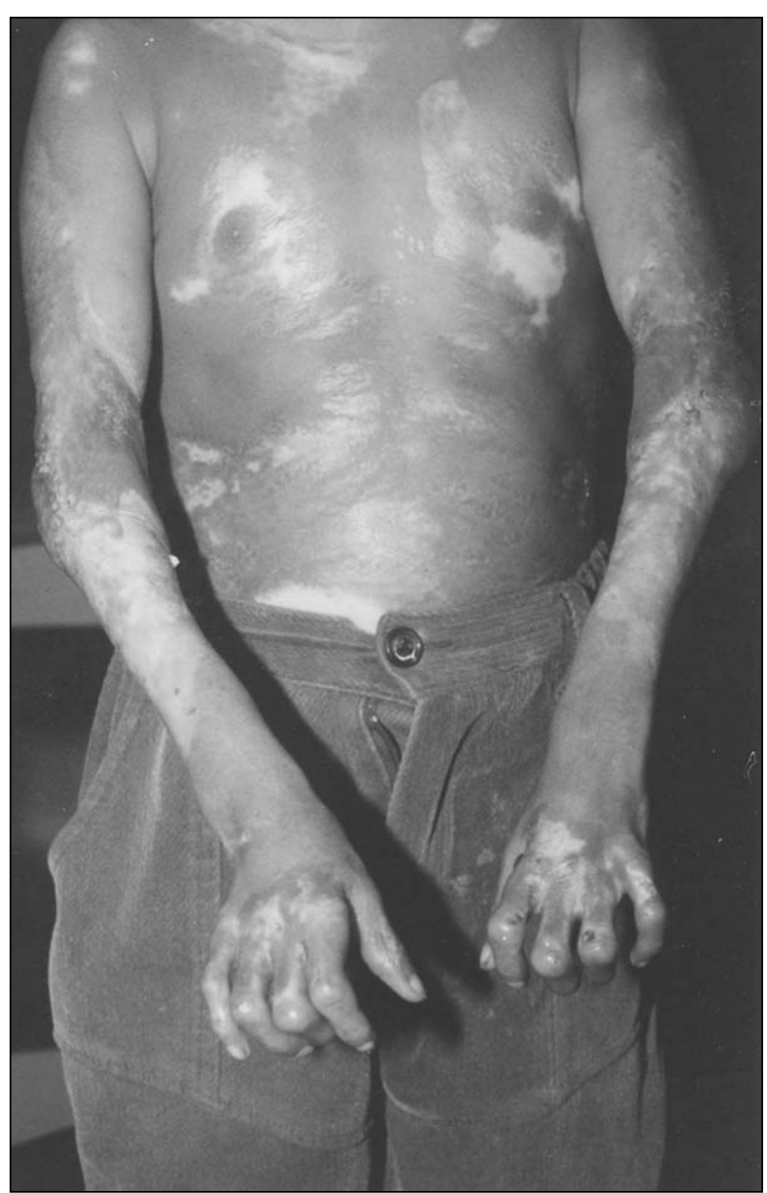

Fig.-3: Fingers having tapered appearance with sclerodactyly

normally functioning kidneys. Barium swallow oesophagus and upper GI endoscopy findings were normal. Echocardiography was normal and color Doppler study suggested normal blood flow in all the major arteries. Skin biopsy was done. It showed thinning of the epidermis and atrophy of the dermal appendages. There were infiltration of lymphocytes and plasma cells.

Considering the history, clinical findings and investigations, $\mathrm{MH}$ was diagnosed as a case of diffuse cutaneous systemic sclerosis (DCSS) with hypothyroidism.

MH was getting thyroxin since last 3 years and tab. Methotrexate was started in a dose of $5 \mathrm{mg} / \mathrm{m}^{2} /$ week before admission. Dose of thyroxin and methotrexate were adjusted and Nifidipine was added as Raynaud's phenomenon did not improve despite of taking local measures. Physical and occupational therapy was advised along with adequate calorie rich diet, vitamins and zinc supplements. The parents were counseled regarding the management and prognosis of the disease and $\mathrm{MH}$ was discharged with the advice of regular follow up. $\mathrm{MH}$ came for follow up on regular basis for up to 1 year after discharge. At the end of one year follow up, he gained 0.5 kilogram weight, skin over the limbs excepting overlying the joints was a little softer. Otherwise he was same as before. After a year MH was lost from follow up.

\section{Discussion:}

Systemic sclerosis is a rare disease with a substantially higher morbidity and mortality than other rheumatic diseases. ${ }^{8}$ It is a multi-system autoimmune rheumatic disorder whose unifying characteristic is the presence of hard skin. ${ }^{6}$ It has a worldwide distribution and affects all races. The role of heredity has not been clarified but examples of familial systemic sclerosis and presence of auto-antibodies in relatives suggests a hereditary predisposition. ${ }^{9}$ Pathogenesis of systemic sclerosis is not clear but the disease process involves immunologic mechanisms, vascular damage and activation of fibroblasts. ${ }^{9}$ Vascular damage precedes fibrosis and involves small arteries, arterioles and capillaries in the skin, GI tract, kidneys, heart and lungs.

Diffuse cutaneous systemic sclerosis (DCSS) usually begins insidiously: the first symptoms are frequently Raynaud's phenomenon and puffy fingers. Early changes include puffiness around the fingers, on the dorsum of the hands and sometimes on the face ${ }^{3}$. An eventual decrease in oedema is associated with tightening of the skin, which spreads proximally from the hands, as was observed in the present case. This tightening and consequent atrophy of the skin gives the characteristic appearance of loss of skin wrinkles and facial expressions, small oral stoma, pursed lips and pinched nose. The hands become shiny with tapered finger ends and tightened skin (Sclerodactyly). Restricted movements produce clawlike deformities, which may also affect the feet ${ }^{2}$. The skin loses hair, oil and sweat glands and so becomes dry and coarse. Ulceration of the affected area may occur. Focal hyper or hypo-pigmented areas may represent a post inflammatory response. ${ }^{1,3}$ The underlying muscles may atrophy and fibrose leading to permanent contractures. 
Pulmonary disease includes arterial and interstitial involvement and can vary from minimal disease to a progressive course that eventually results in right sided heart failure ${ }^{3}$. Scleroderma can also affect other organs. Renal arterial disease can cause chronic or severe episodic hypertension. Oesophageal fibrosis and in-coordination of peristalsis may cause dysphagea and anorexia. ${ }^{3}$ Cardiac fibrosis has been associated with arrhythmia, ventricular hypertrophy and decreased cardiac function. Hypothyroidism occurs in a significant number of patients and may or may not be associated with anti-thyroid antibdies. ${ }^{7}$ According to the American Rheumatology Association, definitive identification of systemic sclerosis requires the presence of the major criterion or documentation of the two minor criteria (Table 1). ${ }^{4}$ MH had major criterion (proximal scleroderma) and also one minor criterion (sclerodactyly). Moreover, he had history of Raynaud's phenomenon, dysphagia and post inflammatory de-pigmented skin areas.

Table-I

Criteria for Diagnosis of Systemic Sclerosis

Major Criterion:

Proximal scleroderma: typical sclerodermatous skin changes (tightness, thickening, and non-pitting induration, excluding localized forms of scleroderma) involving areas proximal to the metacarpophalangeal or metatarsophalangeal joints.

Minor Criteria:

Sclerodactyly: sclerodermatous skin changes limited to digits.

Digital pitting scars resulting from digital ischaemia.

Bibasilar pulmonary fibrosis not attributable to primary lung disease.

There are no specific laboratory tests diagnostic of systemic sclerosis. ${ }^{2}$ In our case, all the investigations were normal excepting mild anemia and previous features of hypothyroidism. Anti scl-70 and antithyroid antibodies were absent, which do not exclude the diagnosis of systemic sclerosis. Anti scl-70 is found only in about $20 \%$ of patients with systemic sclerosis $^{9}$. Anti-thyroid antibodies may not be present in all the cases of systemic sclerosis. ${ }^{9}$
Though x-ray chest of MH was normal but it did not exclude interstitial lung disease. It has been reported that high resolution CT (HRCT) scan detected significant interstitial pulmonary disease in 91\% of children with systemic sclerosis. Majority of them had normal chest radiographs. ${ }^{10}$ HRCT could not be done in our case due to logistic constraints.

There is still no gold standard of therapeutic options for systemic sclerosis. ${ }^{11}$ The main treatment used worldwide for diffuse skin disease for many years had been D-penicillamine but more recently the efficacy of interferon, methotrexate, cyclosporine and azothioprine have been tested. ${ }^{9,12}$ Methotrexate and corticosteroids may be helpful in the early stages of disease. ${ }^{3,13}$ However, corticosteroids later in the course do not appear to be effective and may exacerbate hypertension. ${ }^{13}$

If Raynaud's phenomenon persists despite local measures (keeping hand warm during cold exposure), calcium channels blockers or ACE inhibitors and topical vasodilators may be successful in preventing fingertip ulceractions. ${ }^{3}$ Additional treatment includes physical and occupational therapy to improve flexion contractures and to maintain muscle strength ${ }^{3}$. For treating the major complications like vascular problems, pulmonary hypertension and active fibrosing alveolitis, prostacyclin infusions, oral cyclophosphamides and corticosteroids along with intravenous cyclophosphamides had been trialed with encouraging results ${ }^{12}$. Interesting results have been obtained from autologous bone marrow transplantation in a few paediatric patients of refractory systemic sclerosis. ${ }^{14}$

The course and prognosis of DCSS is poor but it is variable and findings at presentation are not predictive of the outcome. Some patients stabilize after several years, others showing unrelenting progression with death due to end stage pulmonary, cardiac or renal vascular disease. ${ }^{3}$ But there is evidence that clinical course of the disease in children differ from that of adults and has much better outcome ${ }^{15}$.

A standardized treatment protocol for any form of scleroderma is non-existing at the moment. There is no ideal drug available for the treatment of DCSS. 
Symptomatic treatment helps to improve the quality of life. Various disease modifying agents address different pathways of the disease pathogenesis such as vascular disease, autoimmunity and tissue fibrosis. Physiotherapy to maximize the range of motion of all the large joints and psychological counseling are necessary components of the successful management of SSC.

\section{References:}

1. John HT, Eisen AZ. Scleroderma. In: Freederg IM, Eisen AZ, Wolff K, Austen KF, Goldsmith LA, Katz SI, Thomas B, editors. Fitz Patrick's Dermatology in General Medicine. 5th ed. New York: McGraw-Hill; 1999. P.2023-2033.

2. Davidson J, Cleary AG, Bruce C. Disorders of bone, joints and connective tissue. In: McIntosh N, Helms PJ, Smyth RL, editors. Forfar and Arneil's Textbook of Pediatrics. 6th ed. Edinburgh, New York: Churchill Livingstone; 2003. P.1525-1589.

3. Michael LM. Scleroderma. In: Behrman RE, Kliegman RM, Jenson HB, editors. Nelson Textbook of Pediatrics. 17th ed. Philadelphia: W.B. Saunders company; 2004. P. 816-819.

4. Cassidy JT, Petty RE, editors. Textbook of Pediatric Rheumatology. 4th ed. Philadelphia: WB Saunders; 2001.

5. Donald AP. Systemic Sclerosis. e-Medicine 2006 July; Available from http: // www. e-medicine.com
6. Zulian F. Scleroderma in children. Pediatr Clin N Am 2005; 52: 521-545.

7. Kucharz EJ. Thyroid disorders in patients with Progressive systemic sclerosis: A review. Clin Rheumatol 1993; 12: 159-61.

8. Silman AJ. Mortality from scleroderma in England and Wales 1968 -1975. Ann Rheum Dis 1991; 50: 95-96.

9. Bruce CG. Systemic Sclerosis (Scleroderma). In: Braunwald E, Fauci AS, Kasper DL, Hauser SL, Longo DL, Jameson JL, editors. Harrison's Principles of Internal Medicine. 15th Ed. New York: McGraw-Hill; 2001. P.1655-1661.

10. Seely JM, Jones LT, Wallace C, Sherry D, Effmann E. Systemic sclerosis: Using High Resolution CT to detect lung disease in children. Am J Roentgenol 1998; 170:691-697.

11. Foeldvari I, Wulffroat N. Recognition and management of scleroderma in children. Pediatric Drugs 2001; 3: 575-83.

12. Editorial. How can treatment of systemic sclerosis be improved? BMJ 1998; 317: 294-295.

13. Uziel Y, Brian MF, Bernice RK, Rae SMY, Laxer RM. Methotrexate and corticosteroid therapy for pediatric localized scleroderma. J Paediatr 2000;136: 91-95.

14. Lepore L, Kiren V. Autologous bone marrow transplantation versus alternative drugs in pediatric rheumatic diseases. Haematologica 2000; 85: 89-92.

15. Foeldvari I, Zhavania M, Birdi N. Favourable outcome in 135 children with juvenile systemic sclerosis: results of a multinational survey. Rheumatology 2000; 39: 55-69. 